\title{
Estudio del efecto de recubrimientos de sericina extraída de los capullos del gusano de seda Bombyx mori en la degradación de frutas
}

\author{
Giovanni Alberto Cuervo Osorio*; Yesenia Andrea Murillo Arias; Laura Urrea Vélez \\ Programa de Bioingeniería, Facultad de Ingeniería, Universidad de Antioquia (UdeA), \\ Calle 67 carrera 53 - 108, Medellín, Colombia. \\ *giovanni.cuervo@udea.edu.co
}

Fecha recepción: enero 24 de 2020

Fecha aceptación: mayo 30 de 2020

\begin{abstract}
Resumen
Las pérdidas en la producción de frutas luego de la poscosecha son provocadas por descomposición, infestación y ataques microbianos. El uso de recubrimientos permite incrementar el tiempo de conservación de las frutas, estos funcionan como una barrera. En este proyecto se produjeron recubrimientos de sericina extraída del gusano de seda Bombyx mori. Se realizó el proceso de extracción de sericina empleando una solución acuosa de carbonato de sodio y posteriormente se realizó un proceso de diálisis con el fin de eliminar las sales presentes en la solución, se realizaron los recubrimientos empleando diferentes técnicas de impregnación. Se evaluó el efecto de los recubrimientos de sericina en la degradación de fresas y bananos; se tuvo una muestra control tanto para bananos como para fresas, estos controles tuvieron el mismo tratamiento previo pero no se les aplicó ningún recubrimiento, los frutos se impregnaron con capas de la solución de sericina hasta cubrir completamente su superficie, este proceso se hizo de una a cuatro capas. Se almacenaron durante 5 días, se llevó un control diario de la temperatura y la humedad del sitio de experimentación. La técnica por inmersión generó exceso de humedad en la superficie del fruto, generando que el fruto de madura más rápido, y aparecieran diferentes microorganismos, al comparar los dos tipos de técnicas se evidenció que los realizados por pincelado presentan mejores resultados, sin embargo al aumentar el número de capas hubo presencia de agentes patógenos, se comprobó que la sericina es una alternativa para el desarrollo de recubrimientos.
\end{abstract}

Palabras clave: Seda; Sericina; Recubrimientos; Agentes Patógenos; Degradación. 


\title{
Study of the effect of sericin coatings extracted from Bombyx mori silkworm cocoons on fruit degradation
}

\begin{abstract}
Post-harvest fruit production losses are caused by spoilage, infestation and microbial attack. The use of coatings allows to increase the time of conservation of the fruits, these work as a barrier. In this project, sericin coatings extracted from the Bombyx mori silkworm were produced. The process of extraction of sericin was carried out using an aqueous solution of sodium carbonate and later a process of dialysis was carried out in order to eliminate the salts present in the solution, the coatings were made using different techniques of impregnation. The effect of the sericin coatings on the degradation of strawberries and bananas was evaluated; a control sample was taken for both bananas and strawberries, these controls had the same pre-treatment but no coating was applied to them, the fruits were impregnated with layers of the sericin solution until their surface was completely covered, this process was done in one to four layers. They were stored for 5 days, and the temperature and humidity of the experimental site were monitored daily. The technique by immersion generated excess humidity on the surface of the fruit, generating that the fruit ripens faster, and different microorganisms appeared, comparing the two types of techniques it was evident that those made by brushstroke present better results, however when increasing the number of layers there was presence of pathogens, it was verified that the sericin is an alternative for the development of coatings.
\end{abstract}

Keywords: Silk; Sericin; Coatings; Pathogens; Degradation.

\section{Estudo do efeito dos revestimentos de sericina extraídos dos casulos de bicho-da-seda Bombyx mori na degradação dos frutos}

\section{Resumo}

As perdas na produção de frutas após a pós-colheita são causadas por decomposição, infestação e ataques microbianos. O uso de revestimentos permite aumentar a conservação das frutas, os quais funcionam como uma barreira. Neste projeto, os revestimentos de sericina foram produzidos a partir do bicho-da-seda Bombyx mori. O processo de extração de sericina foi realizado com uma solução aquosa de carbonato de sódio e, posteriormente, realizou-se um processo de diálise para eliminar os sais presentes na solução, os revestimentos foram realizados utilizando diferentes técnicas de impregnação. Foi avaliado o efeito dos revestimentos de sericina na degradação de morangos e bananas com uma amostra de controle para cada uma. Esses controles tiveram o mesmo tratamento anterior, mas nenhum revestimento foi aplicado a eles. Os frutos foram impregnados com camadas da solução de sericina até cobrir completamente sua superfície, este processo foi realizado até obter quatro camadas. Eles foram armazenados por 5 dias sendo mantido um controle diário da temperatura e umidade do local experimental. A técnica por imersão gerou excesso de umidade na superfície do fruto, fazendo com que o fruto amadurecesse mais rapidamente e surgissem diferentes microrganismos. Ao comparar esses dois tipos de técnicas, evidenciou-se que os realizados por pulverização apresentam melhores resultados, porém ao aumentar o número de camadas onde houve a presença de patógenos; a sericina foi considerada uma alternativa para o desenvolvimento de revestimentos.

Palavras-chave: Seda; Sericina; Revestimentos; Patógenos; Degradação. 


\section{Introducción}

En el mundo las pérdidas en la producción de frutas y verduras luego de la poscosecha están entre un 25 y $50 \%$, la cual es provocada por factores como la descomposición, infestación por insectos y ataques de microorganismos [1]. Colombia es un país que por sus cambios climáticos genera condiciones perfectas para el cultivo de cualquier tipo de planta, permitiendo exportar un gran número de productos frutales. La mayoría de estos alimentos se ven afectados debido a que las distancias para su exportación generalmente son extensas, lo que implica largos periodos de tiempo de almacenamiento, ocasionando que se maduren muy rápido en el recorrido, provocando daños en los mismos y si llegan en condiciones no óptimas para su consumo son desechados, generando pérdidas significativas para los comercializadores. Por lo cual, es el aspecto físico del producto el que genera la primera impresión al consumidor, siendo así uno de los factores más importantes para la aprobación y la compra del mismo.

El banano y la fresa son frutas que tienen un estado de maduración rápida y son sensibles a diferentes factores en el proceso de poscosecha y de exportación. A causa de estos factores han desarrollado métodos para disminuir la velocidad de maduración, y como solución se propusieron empaques y recubrimientos con diversos materiales, los cuales disminuyen significativamente esta problemática en la etapa de poscosecha. Se han llevado a cabo investigaciones sobre biopolímeros como una alternativa para reducir la cantidad de materiales utilizados habitualmente en la fabricación de los recubrimientos, y para aumentar su tasa de biodegradación como una alternativa para cubrir estas necesidades [2].

Por lo anterior, como opción a un nuevo biomaterial para esta aplicación se tiene la sericina, la cual junto con la fibroína son las principales proteínas constituyentes del capullo del gusano de seda Bombyx mori [3]. Durante las diversas etapas de producción de seda cruda y textil, la sericina se puede recuperar para otros usos. Además, la recuperación de esta reduce el impacto ambiental de la fabricación de seda. La sericina constituye el $25-30 \%$ de la proteína de la seda y envuelve la fibroína [4], es biocompatible, biodegradable, resistente a los rayos UV, resistente a la oxidación, antibacteriano, además tiene retención de humedad, gelificante y capacidad de adherencia [5].

Dentro de las principales aplicaciones de la sericina se ha encontrado su amplio uso en el área biomédica, donde hacen uso de esta proteína como un anticoagulante, también en sistemas de liberación de droga, y para ingeniería de tejidos. Por otra parte, la presencia de gran cantidad de aminoácidos hidrofílicos y su capacidad para retener humedad y formar geles, hace viable su uso en el área cosmética. Así mismo se emplea como biomaterial médico y degradable, polímeros compuestos, biomembranas funcionales, hidrogeles, fibras funcionales y telas. Mizoguchi describió una película delgada de sericina reticulada para su uso como membrana separadora de agua y etanol. En 1978, Miyairi y Sugiura informaron de una película de sericina reticulada con glutaraldehído como agente reticulante para la inmovilización de enzimas [4]. Estudios realizados por Tsubouchi, muestran que la sericina mejora la unión de fibroblastos de piel humana cultivada [6]. En 2007, Pornanong Aramwit evaluó el efecto de la crema de sericina en la curación de heridas en ratas, obteniendo después de un examen histológico después de 15 días de tratamiento una curación completa de la herida [7]. Luego en el 2009 el mismo autor realizó un seguimiento de mediadores inflamatorios inducidos por sericina, obteniendo que promueve el proceso de curación de heridas sin causar inflamación [8].

En este proyecto se realizó un recubrimiento a partir de sericina, que como ya se dijo anteriormente es normalmente desechada de los procesos textiles y además posee diferentes funciones biológicas. Se hace uso de esta con la finalidad de recubrir las frutas como el banano y la fresa, para lograr obtener un mayor tiempo en estado de conservación.

\section{Materiales y métodos}

\section{Extracción de sericina}

Para la extracción de la sericina se utilizaron capullos de gusano de seda Bombyx mori, provenientes de la empresa de sericultura CORSEDA ubicada en el departamento del Cauca, los cuales vienen abiertos y sin larva en su interior. Antes de realizar el proceso de extracción, los capullos fueron seleccionados visualmente, es decir, según su aspecto físico, tamaño y que no presentaran manchas en su interior, lo cual está asociado a productos de desecho de la larva, lo que conlleva a disminuir la pureza de la sericina obtenida.

Para el proceso de desengomado se cortaron los capullos en trozos de aproximadamente un $\mathrm{cm}$, esto con el fin de aumentar la superficie de contacto. Posteriormente se llevó a una solución 
acuosa de agua destilada con carbonato de sodio $\left(\mathrm{Na}_{2} \mathrm{CO}_{3}\right)$ de $99 \%$ de pureza, marca químicos JM en una relación molar 1:1000, de acuerdo con la formulación realizada por E. Echeverri [9].

Para el proceso de desengomado se adiciona el carbonato de sodio a la solución de agua hasta su dilución y se calentó hasta alcanzar una temperatura aproximada de $70^{\circ} \mathrm{C}$. Posteriormente, se incorporaron los capullos de seda previamente cortados, se llevó a agitación manteniendo la temperatura en aproximadamente $70^{\circ} \mathrm{C}$, esta variable se debe controlar para que no se pierda temperatura durante el proceso lo cual alteraría las propiedades de la sericina, llegando al punto de desnaturalizarla, se dejó en agitación durante aproximadamente 2 horas, hasta observar que los trozos de capullo generen una madeja de hilo, y que la solución esté de una tonalidad café traslúcida, transcurrido este tiempo se retiró la madeja de seda, y la solución resultante la cual está compuesta de agua destilada, sericina y carbonato de sodio, se dejó enfriar a temperatura ambiente, este proceso se observa en la figura 1.

\section{Diálisis de la sericina}

Una vez el líquido se enfrió se procedió a realizar un proceso de diálisis, con el fin de retirar de la solución la sal de carbonato de sodio, se empleó una membrana semipermeable de celulosa, la cual permite la difusión de esta sal, la cual fue empleada durante el proceso de desgomado. La solución de sericina se introdujo en la membrana de diálisis, la cual se sumergió en agua destilada, logrando un proceso de intercambio iónico entre la solución de sericina y el agua destilada, el proceso se llevó a cabo durante 2 días, en el primer día se realizó cambios de agua destilada cada 2 horas y en el segundo día este proceso se realizó cada 4 horas. Una vez finalizado el tiempo de diálisis se llevó la solución a calentamiento durante una hora con una temperatura aproximada de 90 a $100^{\circ} \mathrm{C}$, se conoce que la sericina está compuesta de 3 capas, lo que permite este proceso térmico es llegar hasta la capa más interna y obtener una concentración más alta y pura de la sericina [10]. Finalizado este proceso se dejó enfriar la solución a temperatura ambiente.

\section{Concentración}

Para conocer la concentración final de la solución se tomaron $3 \mathrm{ml}$ de la solución y se depositaron en 3 vidrios reloj previamente pesados, posteriormente se llevaron a una estufa a $50^{\circ} \mathrm{C}$ durante 24 horas, con el fin de evaporar el agua y que quedara solo la sericina, finalizado este tiempo se pesó nuevamente el vidrio reloj y se hizo la diferencia con el peso inicial, así se obtuvo una concentración $\%$ W/V.

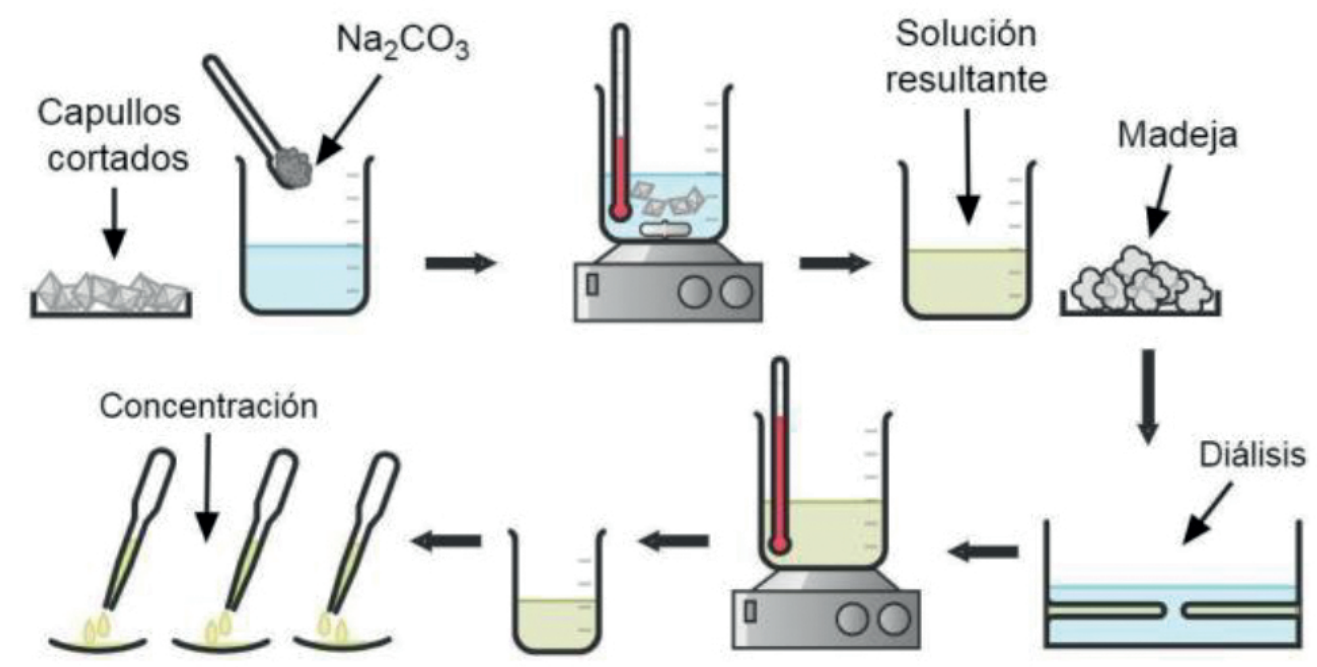

Figura 1. Proceso de extracción, diálisis y dilución de la sericina extraída de los capullos de gusano de seda Bombyx mori. 


\section{Realización de los recubrimientos}

Selección del fruto. Se utilizaron frutos de fresas y bananos, los cuales se obtuvieron de un mercado local (Plaza Minorista José María Villa, Medellín, Colombia). Los frutos fueron transportados al laboratorio de Biomateriales de la Universidad de Antioquia. Para el estudio, se seleccionaron las fresas y bananos con un tamaño uniforme y madurez comercial, descartando visualmente los frutos con daños físicos.

Limpieza y preparación de los frutos. Para la limpieza de las fresas, se pasó por un proceso de lavado con agua destilada por inmersión durante 5 minutos, posteriormente se secaron a temperatura ambiente, para los bananos se cortaron del racimo, conservando el pedicelo. Luego se pasó por un proceso de lavado, primero con agua destilada por inmersión durante 10 minutos, posteriormente se lavaron con hipoclorito de sodio al $0,02 \%$ y finalmente se lavó con agua destilada. Una vez finalizado el proceso de limpieza, se dejaron secar a temperatura ambiente como se observa en figura 2.

Aplicación de los recubrimientos. Una vez lavados los frutos, se dispuso a colgar los bananos del pedicelo con un hilo grueso que resistiera su peso y se organizó de forma separada evitando contacto entre ellos. Mientras que las fresas fueron situadas en una refractaria organizadas también de forma separadas y de manera que el sépalo quedara sobre la refractaria como se muestra en figura 3.

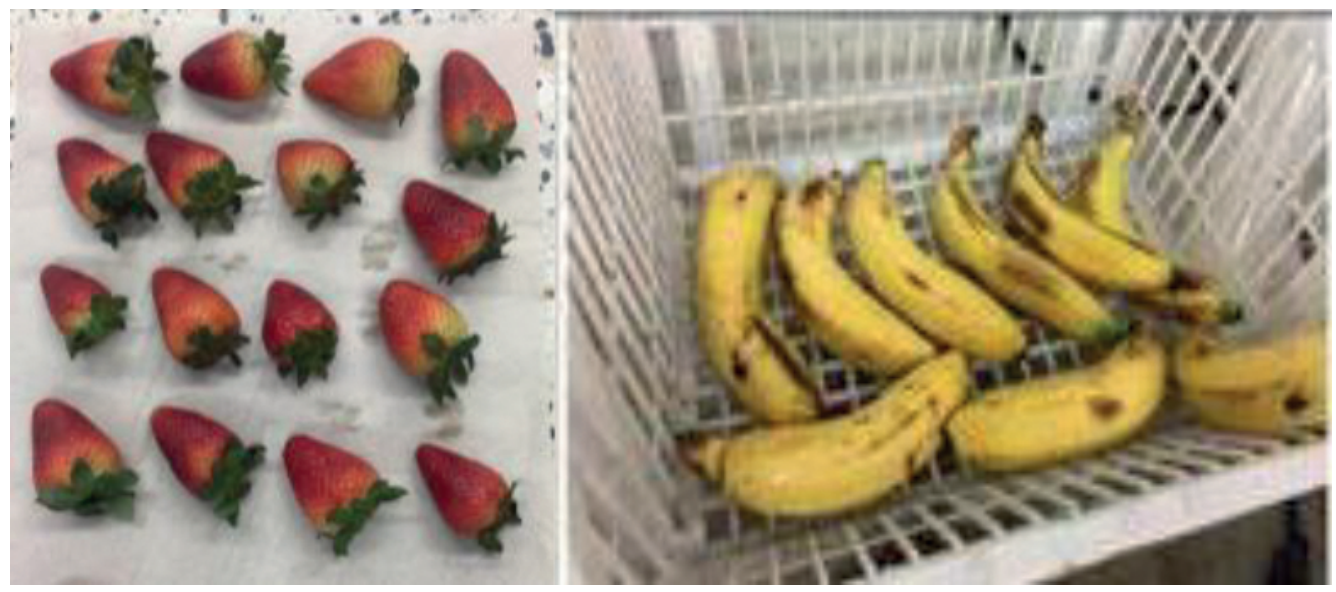

Figura 2. Frutos después de pasar por el proceso de limpieza y preparación.
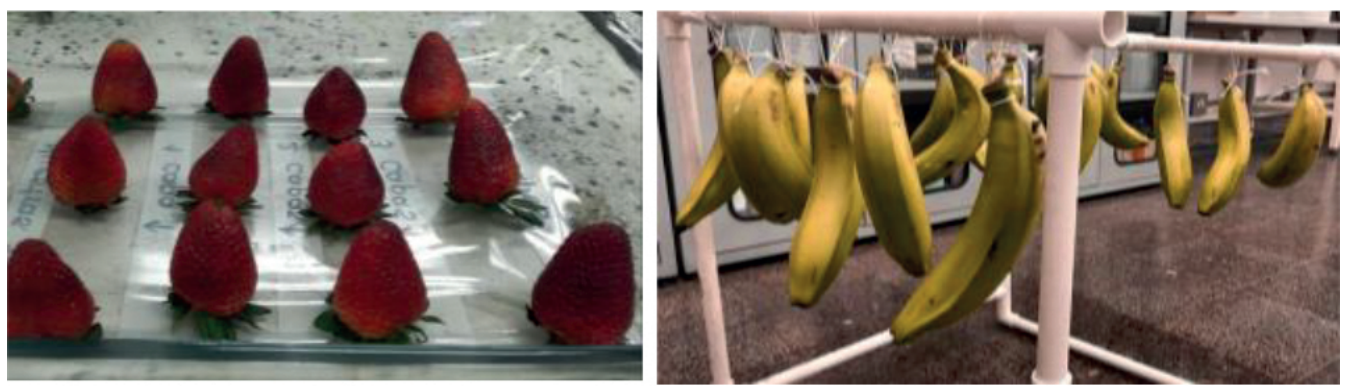

Figura 3. Frutos con las respectivas capas de recubrimientos.

Para recubrir las fresas y bananos se tuvieron en cuenta dos diferentes técnicas, por inmersión y por pincelado. La primera técnica consistió en sumergir los frutos impregnados en su totalidad con la solución de sericina, posteriormente se retiraron y se dejó escurrir el exceso de la solución, para los recubrimientos por pincelado se aplicó a cada fruto, una capa de la solución de sericina hasta cubrir completamente su superficie y se dejaron secar a temperatura ambiente. El proceso se hizo hasta obtener muestras con una, dos, tres y cuatro capas; Además se dejaron tres muestras sin recubrir las cuales se usaría como control. 


\section{Control del proceso}

El control se realizó a temperatura ambiente y se llevó a cabo por 5 días. Diariamente se hizo un registro fotográfico y apuntes sobre los cambios que se lograron evidenciar en las muestras, tales como cambio de color e índice de degradación, también se registró el cambio de humedad y temperatura, mediante un equipo que sensa estas dos variables.

\section{Resultados}

Una vez realizado el proceso de extracción de sericina, se obtuvo las concentraciones de las dos soluciones preparadas para los recubrimientos de las muestras, dichas concentraciones se reportan en la tabla 1.

Tabla 1. Concentraciones de las soluciones de sericina utilizadas para los recubrimientos.

\begin{tabular}{cc}
\hline Recubrimientos & Concentraciones (g/L) \\
\hline Fresas & 9,2 \\
Bananos & 10,4 \\
\hline
\end{tabular}

\section{Control del proceso obtenido para las fresas}

En la figura 4 se hace la comparación al día cuatro entre las dos técnicas usadas, se puede evidenciar un deterioro mayor en la técnica por inmersión, esto debido al exceso de solución que quedó en la fresa luego del recubrimiento, mientras que para las fresas recubiertas por medio de pincelado se puede observar que físicamente tiene un mejor
Se evidencia que obtener soluciones con la misma concentración es un proceso difícil, sin embargo, las soluciones obtenidas no tuvieron una gran diferencia entre ellas.

Para la limpieza de los bananos, al pasarlos por el proceso de lavado con el hipoclorito de sodio al $0,02 \%$, se evidenció un deterioro físico en la cáscara del banano, el cual afectó de manera significativa las propiedades ópticas debido a que adquirió un color más oscuro en gran parte de su superficie. Por otro lado, las fresas no presentaron ningún deterioro visible al limpiarlas. Al emplear el método de aplicación de recubrimientos por inmersión en las fresas, se observó que el fruto quedó con exceso de la solución, lo cual provocó una rápida descomposición, mientras que el método por pincelado proporcionó un recubrimiento en cantidades adecuadas para ambos frutos, si bien por la método de pincelado se obtienen recubrimientos más homogéneos, es una técnica que requiere más tiempo de trabajo.

aspecto, lo que indica que la técnica por pincelado presenta un mejor comportamiento.

A continuación se muestra el seguimiento para las fresas recubiertas por el método de pincelado, esto debido a que como se mencionó anteriormente fue la técnica que presentó los mejores resultados.

Las fresas se dispusieron como se muestra en la tabla 2.

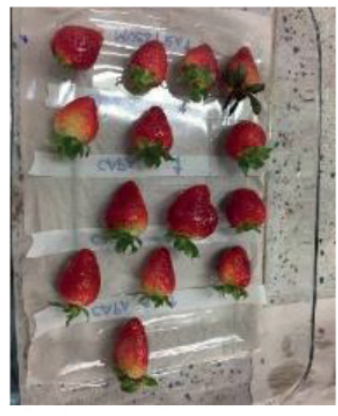

a

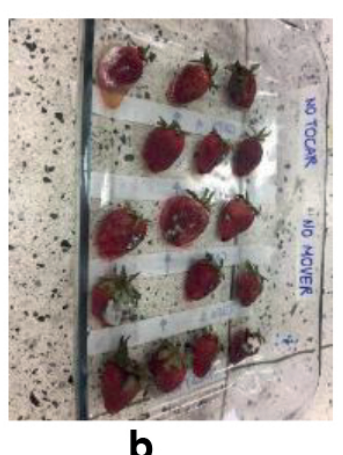

b

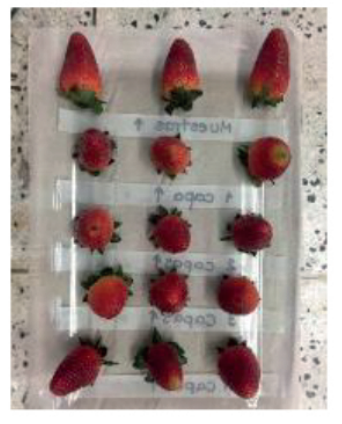

C

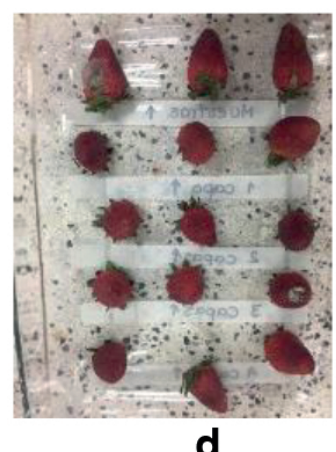

d

Figura 4. Comparación entre técnicas a. día 1 con recubrimiento por inmersión, b. día 4 con recubrimiento por inmersión, c. día 1 con recubrimiento por pincelado d. día 4 con recubrimiento por pincelado. 
Tabla 2. Disposición de las fresas en el ensayo.

\begin{tabular}{cc}
\hline Número de fila & Muestras \\
\hline 1 & Control \\
2 & Con una capa \\
3 & Con dos capas \\
4 & Con tres capas \\
5 & Con cuatro capas \\
\hline
\end{tabular}

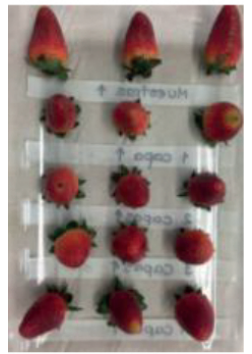

a

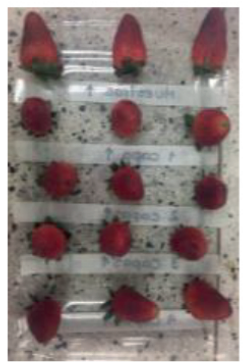

b

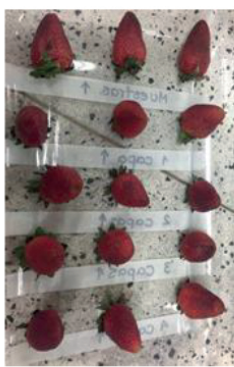

C

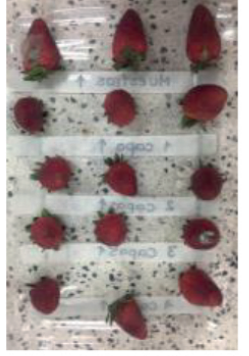

d

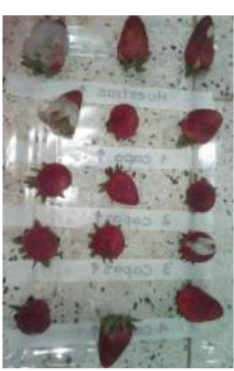

e

Figura 5. Control de los recubrimientos de las fresas. (a) Día 1, (b) Día 2, (c) Día 3, (d) Día 4 y (e) Día 5.

En la figura 5 se observa el seguimiento hecho durante cinco días a las fresas, se puede evidenciar en la figura 5.a, la cual corresponde al día uno, que no hubo ningún cambio visual en los frutos, para el día dos el cual se visualiza en la figura 5.b, empieza a aparecer un cambio en la coloración de las fresas tornándose más oscuras, sin embargo las muestras con una capa de recubrimiento, esto se presentó en menor manera, en el tercer día (figura 5c), se apreció el crecimiento de moho gris en una muestra control como se señala en la imagen, esto indica la aparición de un agente patógenos. Esto también sucedió en una de las muestras con una capa de recubrimiento, para este día se observó que las muestras control y las que tenían 4 capas de recubrimiento tenían una descomposición leve, cabe resaltar que las de una sola capa de recubrimiento presentaba una mejor conservación que el resto de muestras, para el día cuatro el cual se observa en la figura 5.d, se evidencia que aumentó la presencia del moho gris o agente patógeno, además una nueva muestra control se vio afectada por el mismo moho gris, en una de las muestras de tres capas de recubrimiento se aprecia una zona de descomposición del fruto, en donde se observa la presencia de un agente patógeno, para las muestras con cuatros capas en este día aún no hay presencia de aparición de microorganismos en su superficie, sin embargo visualmente sus calidad no era la mejor, esto debido a que se tornaron de una tonalidad oscura en algunas zonas. Por último, para el día cinco correspondiente a la figura 5.e, las muestras de dos y cuatro capas de recubrimiento presentaron la aparición de un agente patógeno, esto también sucedió en una segunda muestra de una capa de recubrimiento, lo anterior indica que los frutos con dos y cuatro capas tuvieron mayor tiempo sin la aparición de agentes patógenos, sin embargo visualmente la muestras con una sola capa de recubrimiento tienen mejor apariencia, y una de sus muestras no registro aparición de microorganismos en sus superficie, y una segunda muestra se conservó hasta el día cinco en buenas condiciones.

Control del proceso obtenido para los bananos

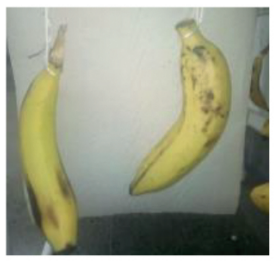

a

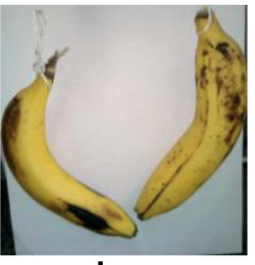

b

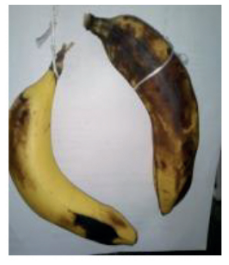

C
Figura 6. Control de muestras a. muestra control día 1, b. muestra control día 3, c. muestra control día 5. 


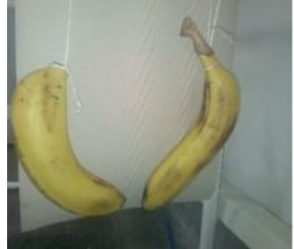

a

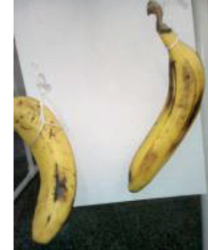

b

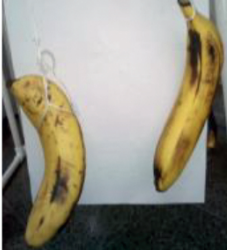

C

Figura 7. Control de muestras con una capa de recubrimiento, a. muestra día 1, b. muestra día 3, c. muestra día 5.

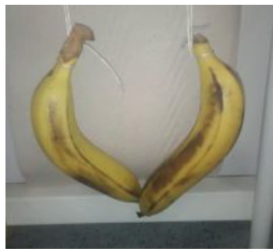

a

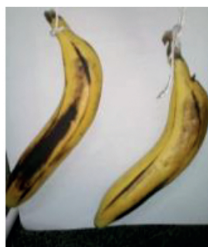

b

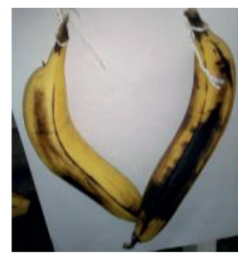

c

Figura 8. Control de muestras con dos capas de recubrimiento, a. muestra día 1, b. muestra día 3, c. muestra día 5.

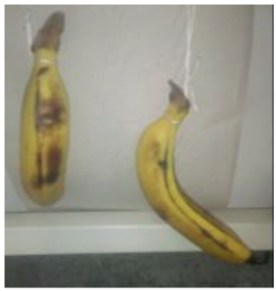

a

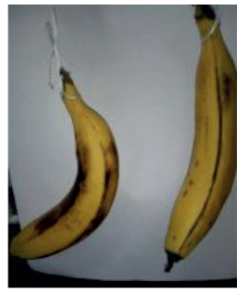

b

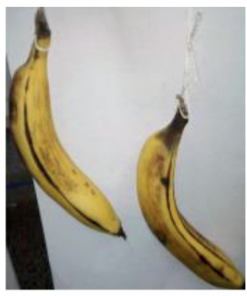

C

Figura 9. Control de muestras con tres capas de recubrimiento, a. muestra día 1, b. muestra día 3, c. muestra día 5.

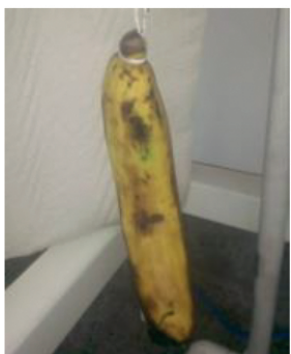

a

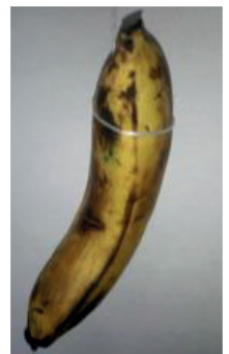

b

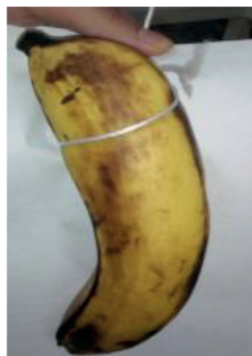

C

Figura 10. Control de muestras con cuatro capas de recubrimiento, a. muestra día 1, b. muestra día 3, c. muestra día 5.

En las figuras 6 a la 10, se muestra el proceso de control que se le realizó a los bananos, este registro control se hizo durante 5 días, tomando un registro fotográfico los días 1, 3 y 5 respectivamente, desde el primer día para todas las muestras se evidenció un color marrón en la cáscara de los frutos, lo cual se cree es debido al proceso de limpieza, tanto para el control como para las muestras con recubrimiento, se apreció que esta mancha creció con el transcurso de los días, al comparar las muestras en el último día de control fue evidente que los frutos con recubrimientos (figura 7.c,8.c,9.c y 10.c) tenían mayor firmeza en su cáscara, además que lograban en comparación con la muestra control (figura 6.c) disminuir la extensión de la mancha en su superficie. Solo una muestra correspondiente al fruto recubierto con dos capas (figura 8.c), presentó una afectación considerable en su superficie, sin embargo este problema estaba presente desde el día uno (figura 8.a) y al observar el comportamiento de la zona de afectación de la muestra control con el transcurso de los días, se puede presumir que el recubrimiento atenuó la propagación de la mancha en la cáscara, esto indica que los recubrimiento podrían tener la capacidad de disminuir el proceso de degradación de los bananos.

El control de humedad y temperatura registraron los valores que se presentan en las tablas 3 y 4 . 
Tabla 3. Control de temperatura

\begin{tabular}{ccccc}
\hline Hora & Recuento & Promedio & Desviación estándar & Coeficiente de variación \\
\hline 10:00 & 18 & 23,7778 & 1,00326 & $4,21933 \%$ \\
12:00 & 18 & 24,4444 & 0,615699 & $2,51877 \%$ \\
$17: 00$ & 18 & 25,6667 & 0,840168 & $3,27338 \%$ \\
Total & 54 & 24,6296 & 1,13763 & $4,61895 \%$ \\
\hline \multicolumn{5}{c}{ Tabla 4. Control de humedad } \\
\hline Hora & Recuento & Promedio & Desviación estándar & Coeficiente de variación \\
\hline 10:00 & 18 & 37,9444 & 2,12747 & $5,60681 \%$ \\
12:00 & 18 & 36,0 & 1,37199 & $3,81108 \%$ \\
17:00 & 18 & 36,3889 & 3,0125 & $8,27863 \%$ \\
Total & 54 & 36,7778 & 2,38444 & $6,48336 \%$ \\
\hline
\end{tabular}

De la tabla 3 y 4 se puede observar un coeficiente de variación para cada hora menor al $15 \%$, lo cual indica que los datos tienen poca dispersión entre ellos, además podemos observar que la mayor temperatura registrada en la tabla 3 se presenta a las cinco de la tarde y la menor se registra a las diez de la mañana, mientras que la mayor humedad registrada en la tabla 4 se presenta a las diez de la mañana y la menor se registra a las doce de la tarde. Se puede evidenciar que no existe una diferencia estadística significativa para las dos variables, por lo que se puede afirmar que los cambios producidos en los frutos no se deben a un cambio de temperatura o humedad.

\section{Discusión}

De acuerdo con los resultados obtenidos, al realizar la extracción y concentración de las soluciones de sericina, se evidencia tienen una leve diferencia en su porcentaje de concentración, esto se debe a que los gusanos de seda según la época del año varían las propiedades de los hilos de seda, esa decir las concentraciones tanto de fibroína como de sericina se ven afectados por este motivo, también se puede atribuir a que en el proceso de desengomado los capullos del gusano de seda no se homogenizan completamente en toda la solución acuosa de carbonato de sodio, puesto que el control de la temperatura durante el proceso es difícil de mantener constante, además de que el tiempo que se emplea difiere, dado que en ocasiones la dilución de los capullos es más rápida que en otras, es debido a que la solución se puede saturar, por lo cual la homogeneización no se logra completamente. Lo anterior da lugar a que las soluciones preparadas difieran en el valor de su concentración final.

Al comparar las dos técnicas usadas, inmersión y pincelado respectivamente, se pudo evidenciar que por inmersión se logra cubrir mejor el fruto, sin embargo esta técnica genera excesos de la solución en la superficie del fruto, lo que influencia a la aparición de agentes patógenos sobre el fruto, se logró observar que la técnica por pincelado elimina el problema de exceso de solución, sin embargo es una técnica compleja y que requiere de mayor tiempo para su ejecución, no obstante se evidenció que esta técnica mejora las propiedades del recubrimiento.

Al observar las fresas se logró apreciar que mientras más capas de recubrimiento tuvieran, se volvía de un color más oscuro esto se atribuye a que se disminuye la permeabilidad del fruto, por lo cual no se da un intercambio iónico del fruto con el medio, lo que ocasiona que se de este fenómeno [11]. Durante el proceso de control se observan fresas con presencia de microorganismos patógenos, por sus características físicas (moho gris) se cree que pueda tratarse del hongo Botrytis cinerea, este hongo es difícil de detectar durante la cosecha de los frutos debido a que casi siempre se comporta como hongo inactivo (latente), por lo cual se infiere que para el tercer día de control, el fruto con una capa de recubrimiento que presenta el crecimiento de este microorganismo sobre su superficie ya se encontraba infectado desde antes de empezar el registro, solo que se encontraba latente y se logró activar al lograr las condiciones óptimas para su crecimiento, esto puede traer 
como consecuencia la infestación de las demás fresas [12]. Por otro lado, la muestra con tres capas de recubrimiento que empezó a presentar mayor grado de descomposición es por la posible presencia del hongo Rhizopus Stolonifer, el cual es el causante de la descomposición en estos frutos, por esta razón se observaron dichas alteraciones en las muestras, unas con un nivel afectación más leve que otros [13]. Si bien se puede afirmar que hay presencia de agentes patógenos que afectan a las fresas, se debe realizar un ensayo que permita la identificación de hongos y así confirmar que tipo de hongos se presentaron en el fruto, sin embargo es notorio en algunos frutos que no hubo presencia de microorganismos en su superficie.

Al realizar el análisis de los bananos, se pudo observar que la cáscara tanto para los de control como los de prueba, desde el primer día no presentan un aspecto visual óptimo, debido a que, en el proceso de limpieza y preparación del fruto, el porcentaje de hipoclorito de sodio empleado a pesar de ser relativamente bajo, ocasiona un ennegrecimiento de la cáscara, esto está relacionado con el grado de madurez del fruto, por lo que puede ser más susceptible a daños por esta solución. Lo anterior se ratifica con el hecho de que los bananos liberan enzimas de polifenol oxidasa causantes del color marrón o negro de la cáscara de la fruta, el exceso de hipoclorito provoca una aceleración en ese proceso causando daños superficiales de estos frutos [14]. Sin embargo, se presume que los recubrimientos tienen la capacidad de disminuir el proceso de degradación de los frutos, debido a que al observar el último para todas las muestras se evidenció que en los frutos con recubrimiento de sericina se disminuye la propagación de las manchas, lo cual no pasó en el fruto control el cual al quinto día estaba totalmente negro en su superficie.

Los recubrimientos realizados demostraron tener una leve capacidad protectora de los frutos contra los diferentes agentes patógenos que se presentan en estos frutos, cabe resaltar que la técnica que mejor funcionó fue por pincelado con una sola capa de recubrimiento para las fresas, si bien algunos presentaron la aparición de dichos microorganismos se debe tener en cuenta diferentes factores para esto, como lo son el tipo de técnica de recubrimiento, la concentración de la soluciones de sericina las cuales se pueden aumentar, sin sobrepasar el punto de gelificación de esta proteína, y así tener mayor cantidad de sericina en la solución final.
El seguimiento de la humedad y la temperatura del lugar de ensayo, permitió observar que estas variables eran constantes en el tiempo, puesto que no se tuvo una diferencia estadística significativa entre las diferentes horas de medición, lo que indica que los frutos no sufrieron alteraciones debido a estas variables.

\section{Conclusiones}

Para mejorar la concentración final de sericina se debe controlar diferentes variables como la temperatura, tiempo de agitación de la solución, tiempo de vaciado de agentes químicos y cantidad de agregación de capullos de seda cortados. Además, se debe tener un control riguroso para la elección del fruto del ensayo puesto que si no son obtenidos del mismo lote se puede alterar el resultado, además, que en algunos puede aparecer infestación por agentes patógenos latentes en el fruto.

El método de pincelado fue el más adecuado en cuanto al control de humedad, evitando así frutos con exceso de la solución acuosa de sericina y disminuyendo visualmente la proliferación de agentes patógenos. Las fresas recubiertas con la solución de sericina evidenciaron una reducción de población microbiana en comparación con las muestras control que no se les aplicó recubrimiento. Las fresas con una capa de recubrimiento fueron las que tuvieron mejores resultados en la aparición de agentes patógenos. Los recubrimientos de sericina aplicados en la superficie de los bananos evidenciaron influir sobre el proceso de degradación de los frutos. Finalmente, se observó que al aumentar el número de capas de recubrimiento a los frutos, disminuye el intercambio iónico que tienen estos con el medio, causando así que se tornen con una apariencia más opaca.

\section{Referencias bibliográficas}

[1] Yahia EM, Ariza-Flores R. Tratamientos físicos en poscosecha de fruta y hortaliza. Rev. Horticultura. 2001:80-89.

[2] Moncayo Martinez DC. Desarrollo de un recubrimiento comestible a partir de un biopolímero para prolongar la vida útil de frutas frescas (Tesis de maestría). Bogotá, Colombia: Universidad Nacional de Colombia; 2013.

[3] Aiko H. Gusanos de seda modificados genéticamente para desarrollar medicamentos. 
2013. [Enlinea]. Available: https://www.nippon. com/es/features/c00508/. Acceso 13 de marzo 2018.

[4] Zhang YQ. Applications of natural silk protein sericin in biomaterials. 2002;20(2):91-100.

[5] Roy Choudhury AK. Finishes for protection against microbial, insect and UV radiation. in Principles of Textile Finishing, 2017.

[6] Tsubouchi K, Igarashi Y, Takasu Y, Yamada $\mathrm{H}$. Sericin enhances attachment of cultured human skin fibroblasts. Biosci., Biotechnol., Biochem. 2005;69(2):403-405.

[7] Aramwit P, Sangcakul A. The effects of sericin cream on wound healing in rats. Biosci. Biotechnol. Biochem, 2007.

[8] Aramwit P, Kanokpanont S, De-Eknamkul W, Srichana T. Monitoring of inflammatory mediators induced by silk sericin. J. Biosci. Bioeng. 2009;107(5):556-561.

[9] Correa EE, Lopera DOG, Restrepo SG, OssaOrozco CP. Effective sericin-fibroin separation from Bombyx mori silkworms fibers and low- cost salt removal from fibroin solution. Rev. Fac. Ing., Univ. Antioquia. 2020;(94):97-101.

[10] Miguel GA. Extracción de sericina proveniente del proceso de desengomado de la seda, para su aprovechamiento como ingrediente para el desarrollo de alimentos funcionales, 2014: 57.

[11] Lopez CA.Caracterización de sericina obtenida a partir de aguas de desengomado de seda natural. Prospectiva. 2013;11(2):7-12.

[12] Koike T, Mark B. El Moho Gris o Pudrición de Fresa. United States: California Strawberry Comission; 2016.

[13]Fraire Cordero ML. Hongos Patógenos en Fruto de Fresa (Fragaria $x$ ananassa Duch.) en Postcosecha. Rev. Mex. fitopatol. 2003;21(3):285-291.

[14]Mosquera Sánchez S, Barco Hernández P, Burbano Delgado A, Medina M, Villada Castillo $\mathrm{H}$. Efecto de recubrimiento natural y cera comercial sobre la maduración del banano (musa sapientum). Biotecnol. Sect. Agropecu. Agroind. 2009;7(2):70-76. 